\title{
Neural Network Models for Traffic Noise Quality Prediction: A Comparative Study
}

\author{
Nayef Al-Mutairi* and Al-Rukaibi F
}

Department of Civil Engineering, College of Engineering and Petroleum, Kuwait University, P.O. Box 5969, Safat 13060, Kuwait

\begin{abstract}
Problem statement: In the absence of long-range strategic plans, the urban infrastructural growth in Kuwait has been accompanied with significant adverse impacts on the urban environment, and has resulted in the deterioration of the quality of urban life. With this continuous growth, a growing percentage of urban population will be adversely affected by traffic noise pollution.

Approach: Traffic-generated noise pollution was monitored at nearly four roadway locations in four districts in metropolitan Kuwait in 2007-2008. At each district, a sample of freeway, arterial, collector, and local residential streets were included in the noise and traffic flow monitoring plan. In addition to the analysis of noise, flow, and their interrelationships, three models - two neural network models and one regression model, were employed to predict traffic noise pollutions.

Results: Five uncorrelated components of the noise pollution were used as the ANN model input to predict noise pollution using a back propagation neural network (BPNN), general regression neural network (GRNN) algorithm and a general regression model. The model inputs were the number of vehicles, the equivalent number of cars per hour, the heavy vehicle percentage, the width of road and the average height of buildings facing the road. The models optimum architectures were determined for BPNN model by varying the number of hidden layers, hidden transfer function, test set size percentages, and initial weights.

Conclusion: Findings indicate that traffic noise is at or above, the standard outdoor limits in most locations, and especially at arterial roadways and freeways. Comparison of the two prediction results showed that GRNN had the ability to calibrate the multi-component traffic noise and yield reliable results close to that by direct measurements. It was concluded that the optimal BPNN model used in this study provided reasonable predictions of noise profiles for all the data sets employed in this study, with two parallel hidden layer back-propagation showing the best overall prediction. This research has demonstrated the great potential of GRNN modeling technique over BPNN techniques in predicting traffic noise.
\end{abstract}

Keywords: Traffic Noise; Residential; Kuwait; Noise pollution; Prediction; Artificial neural networks; Noise; Outdoor noise; Noise modeling

\section{Introduction}

The Environmental Impact Statement (EIS), a newly requirement of any proposed development project in the State of Kuwait addresses noise pollutions. The EIS calls for the identification and determination of the magnitude of noise, air, water, land, wetland, and other potential environmental impacts of a new construction project. A variety of models were employed to determine the noise impacts of a development project. Regression models - regressing traffic noise levels on such a contributory variables as traffic volume, mix, and speed, have long been utilized to forecast noise. More recently, the US traffic noise model (FHWA, TNM), is being used across the United States FHWA, 2004 to predict noise impacts of any proposed roadway expansion/ construction [1]. Noise models were generally employed to determine the impact of proposed infrastructural developments on the level of noise pollution, during and after infrastructures are implemented [2].

In addition, Artificial Neural Networks (ANN) can be used to determine the noise impact. They are comprised of a number of "processing units" that are linked via weighted interconnections. ANN is very sophisticated modeling techniques capable of modeling extremely complex functions. In particular, neural networks user gather representative data, and then invokes training algorithms to automatically learn the structure of the data. They are also applicable in virtually every situation in which a relationship between the predictor variables and predicted variables exists even when that relationship is very complex and not easy to articulate in the usual terms of correlations between groups [3-6].
Back propagation neural network (BPNN) is the best known training algorithm and the most useful for neural networks. Devised initially by Werbos [7], BPNN algorithm has lower memory requirements than most algorithms, and usually reaches an acceptable error level quite quickly. A simple network has a feed-forward structure: signals flow from inputs, forwards through any hidden units, eventually reaching the output units [8]. When the network is executed, the input variable values are placed in the input units, and then the hidden and output layer units are progressively executed. Each of them calculates its activation value by taking the weighted sum of the outputs of the units in the preceding layer, and subtracting the threshold. If the network is properly trained, it has then learned to model the function that relates the input variables to the output variables, and can subsequently be used to make predictions where the output is not known [9].

On the other hand, general regression neural network (GRNN) is based on established statistical principles and is capable of functioning

*Corresponding author: Nayef Al-Mutairi, Civil Engineering Department College of Engineering and Petroleum, Kuwait University, P.O. Box 5969, Safat 13060, Kuwait, Tel: +965-481-1188/ext: 5736; Fax: +965-481-7524; E-mail: nayef.almutairi@ku.edu.kw

Received November 03, 2011; Accepted January 16, 2012; Published January 18, 2012

Citation: Al-Mutairi N, Al-Rukaibi F (2012) Neural Network Models for Traffic Noise Quality Prediction: A Comparative Study. J Civil Environment Engg 2:106 doi:10.4172/2165-784X.1000106

Copyright: (c) 2012 Al-Mutairi N, et al. This is an open-access article distributed under the terms of the Creative Commons Attribution License, which permits unrestricted use, distribution, and reproduction in any medium, provided the original author and source are credited. 
after only a few training patterns have been entered [10-12]. Unlike back propagation networks which propagate training patterns through the network many times seeking a lower mean absolute error between the networks output and the actual output, GRNN training patterns are only presented to the network one time. GRNN avoids well-known disadvantages of common feed-forward networks such as: a) The architecture and all weights are completely determined; b) No need for learning and momentum rate; c) The only free parameters to be trained are the smoothing factor. There are several different training procedures for the GRNN. They range from simple iterative to genetic algorithms [13].

The objective of the study was to investigate the uses of an Artificial Neural Networks (BPNN and GRNN algorithms) to predict traffic noise.

\section{Materials and Methods}

The Bruel \& Kjear [14] sound level meter was utilized to measure and record noise levels. Noise monitoring locations were selected such that noise from other sources was negligible. Statistical Analysis Softwares (SAS) were employed to process and analyze the data [15]. Since meteorological conditions such as wind speed and direction, air temperature and relative humidity have considerable effects on noise levels, these parameters were measured at the same time. The minimum measuring time at each measurement point was $5 \mathrm{~min}$. Noise level measurements were obtained with a HD 9019 sound level meter class 1 according to IEC 651 and HD 9102 calibrator for sound level meters type 2 - IEC 942-1988, BS 7189, ANSI S1, 40-1984, a half inch condenser microphone and a tripod.

The neural network that was used for training and prediction was implemented on a commercially available simulation package, NeuroShell ${ }^{\mathbb{} 2}$ (Word System Group, Inc., Mass., USA 1996). The nodes of a neural network are organized into groups, forming layers in the network. The model inputs were the number of vehicles, the equivalent number of cars per hour, the heavy vehicle percentage, the width of road and the average height of buildings facing the road. The input layer is used to distribute the data from the environment to the other layers of the network, which process the data. The output layer passes the network's result back to the environment. The general structure of an ANN is well known and can be found in numerous publications [16-18]. Basically, the learning in the network is achieved through an iterative algorithm that minimizes the mean-absolute errors between the desired and actual outputs [19].

On the other hand, a typical GRNN net is composed of a single hidden layer that contains one hidden neuron for each training pattern. The training set consists of values for $x$, each with a corresponding value for $y$. This regression method produces the estimated value of $y$, which minimizes the mean absolute error. In GRNN network, a hidden layer neuron is created to hold the input vector. The weight between the newly created hidden neuron and the output neuron is assigned the target value. GRNN is based on the following formula:

Expected (output) $=\frac{\int y f(x, y) d y}{\int f(x, y) d y}$

$y=$ output of the estimator,

$x=$ the estimator input vector,

Expected (output) $=$ the expected value of output, given the input vector $\mathrm{x}$,

$f(x, y)=$ the joint probability density function of $x$ and $y$.

Inside each neuron a weighted sum of the inputs is calculated, and this value, called net, is transformed by a bipolar sigmoid or Gaussian functions. The transformed result is sent to neurons in the next layer.

\begin{tabular}{|c|c|c|c|c|c|c|}
\hline & $\begin{array}{l}\text { Speed of vehicles } \\
(\mathrm{Km} / \mathrm{hr})\end{array}$ & $\begin{array}{l}\text { the equivalent number } \\
\text { of cars per hour }\end{array}$ & $\begin{array}{l}\text { the heavy vehicle } \\
\text { percentage }\end{array}$ & $\begin{array}{l}\text { the width of road } \\
(\mathrm{m})\end{array}$ & $\begin{array}{l}\text { average height of buildings } \\
\text { facing the road }(\mathrm{m})\end{array}$ & $\begin{array}{l}\text { Noise levels } \\
L_{e q}(d B A)\end{array}$ \\
\hline ANN Variable Type & Input & Input & Input & Input & Input & Output \\
\hline Mean & 78.5 & 1400 & 0.19 & 6 & 30 & 78 \\
\hline Std. Deviation & 35.9 & 790 & 0.08 & 1.5 & 10 & 11 \\
\hline $25 \%$ percentile & 63.2 & 512 & 0.05 & 2.5 & 15 & 57 \\
\hline $50 \%$ percentile & 72.9 & 1200 & 0.1 & 3.9 & 25 & 72 \\
\hline $75 \%$ percentile & 89.5 & 1580 & 0.25 & 8.5 & 40 & 74 \\
\hline $90 \%$ percentile & 125 & 1710 & 0.3 & 9.1 & 42 & 87 \\
\hline
\end{tabular}

Table 1: Descriptive statistics of the experimental data.

\begin{tabular}{|l|l|l|l|l|l|l|l|}
\hline \multirow{2}{*}{ Roadway } & No. of Lanes & Speed $(\mathrm{Km} / \mathrm{hr})$ & & \multicolumn{3}{c|}{ Traffic Volume $(\mathrm{vph})$} \\
\cline { 2 - 6 } & per Dir. & Mean & Small & Medium & Heavy & Motor Cycle \\
\hline Gulf Road & 3 & $82.1 \pm 16$ & 1328 & 240 & 4 & 0 \\
\hline Minawer Street & 2 & $34.7 \pm 7$ & 1104 & 152 & 52 & 0 \\
\hline Salem Al-Mubarak Street & 2 & $42.5 \pm 10$ & 456 & 96 & 12 & 0 \\
\hline Ghazali Expressway & 3 & $86.1 \pm 19$ & 1204 & 206 & 1308 & 72 \\
\hline
\end{tabular}

Table 2: Study Roadways and Traffic Flow Variables (Typical Peak Hour).

\begin{tabular}{|c|c|c|c|c|}
\hline Model \# & BPNN Architecture & Optimum Test Set \% & $\mathrm{MAE}_{\mathrm{T}}$ & $\mathrm{MAE}_{\mathrm{p}}$ \\
\hline 1 & One hidden layer & 20 & 9.6 & 5.7 \\
\hline 2 & Two hidden layers in series & 10 & 10.1 & 8.1 \\
\hline 3 & Three hidden layers in series & 30 & 5.1 & 11 \\
\hline 4 & Two parallel hidden layers & 10 & 2 & 5 \\
\hline 5 & Three parallel hidden layers & 15 & 7 & 12 \\
\hline 6 & Two parallel hidden layers with a jump connection & 35 & 8 & 6 \\
\hline
\end{tabular}

Table 3: Comparative mean absolute error for trained and prediction BPNN. Architectures at 10,000 training epochs. 


\begin{tabular}{|l|l|l|}
\hline Initial weights & $\mathrm{MAE}_{\mathrm{T}}$ & $\mathrm{MAE}_{\mathrm{P}}$ \\
\hline 0.05 & 4.3 & 7.3 \\
\hline 0.1 & 4.7 & 6.9 \\
\hline 0.2 & 4.8 & 7.5 \\
\hline 0.3 & 4.0 & 5 \\
\hline 0.5 & 8.0 & 11.0 \\
\hline 0.9 & 4.8 & 8.0 \\
\hline 1 & 5.9 & 8.6 \\
\hline 1.5 & 6.7 & 8.9 \\
\hline 2 & 5.3 & 7.8 \\
\hline
\end{tabular}

Table 4: Effect of initial weights on MAE for both training and testing set $\left(\mathrm{MAE}_{\mathrm{T}}\right)$ and prediction set $\left(\mathrm{MAE}_{\mathrm{p}}\right)$.

\begin{tabular}{|l|l|}
\hline Architecture & $6-2-1$ feed forward \\
\hline Input layer transfer function & Linear $[-1,1]$ \\
\hline Hidden layer and output transfer function & Logistic \\
\hline Number of hidden layer & Two parallel layers \\
\hline Number of neurons in hidden layer & 30 \\
\hline Learning rate & 0.1 \\
\hline Momentum rate & 0.3 \\
\hline No. of Epochs & 10,000 \\
\hline
\end{tabular}

Table 5: Optimized parameters used for construction of a back propagation ANN model.

\begin{tabular}{|l|l|l|l|l|l|}
\hline Neural Network Architecture & $\mathrm{MAE}_{\mathrm{T}}$ & $\mathrm{R}_{\mathrm{T}}^{2}$ & $\mathrm{MAE}_{\mathrm{P}}$ & $\mathrm{R}_{\mathrm{P}}^{2}$ & Smoothing factor \\
\hline GRNN with Genetic adaptive & 1.0 & 0.96 & 3.0 & 0.93 & 0.099 \\
\hline GRNN with Iterative adaptive & 3.4 & 0.77 & 9.8 & 0.13 & 0.066 \\
\hline Optimum BPNN & 5 & 0.83 & 4 & 0.87 & \\
\hline Regression & 7.4 & 0.62 & 12.1 & 0.65 & \\
\hline
\end{tabular}

Table 6: Comparison of the neural network performance.

Usually the sigmoid function and the Gaussian function are defined in equation 2 and 3 respectively:

$$
\begin{aligned}
& \mathrm{F}(\text { net })=\frac{1}{\left(1+e^{(-x)}\right)} \\
& \mathrm{F}(\text { net })=\left(\mathrm{e}\left(-\mathrm{x}^{2}\right)\right)
\end{aligned}
$$

This function tends to reduce the effect of outliers. The error of the network is computed by finding the mean absolute error (MAE) and the coefficient of multiple determinations $\left(\mathrm{R}^{2}\right)$ as shown in equation 4 and 5. The error is summed over each pattern, $p$, in the training and prediction set. $\mathrm{O}^{\mathrm{P}}$ is the actual output for each pattern in the set, $\mathrm{O}_{n}^{\mathrm{P}}$ is the output of the network for each pattern, $\mathrm{O}_{m}^{\mathrm{P}}$ is the mean of the output of the network.

$$
\begin{aligned}
& \text { MAE }=\frac{\left|\sum\left(\mathrm{O}_{\mathrm{a}}^{p}-O_{n}^{p}\right)\right|}{\mathrm{N}} \\
& \mathrm{R}^{2}=1-\frac{\sum\left(O_{a}^{p}-O_{n}^{p}\right)}{\sum\left(O_{a}^{p}-O_{m}^{p}\right)}
\end{aligned}
$$

During the adaptation phase, the training algorithm receives part of the data (inputs and outputs) and automatically develops the ANN model. After development, the model can generate the appropriate biological activity performance responses for simulations with varying levels data input. When the learning is complete, the neural network is used for prediction.

\section{Results}

The mean, the standard deviation and the descriptive statistics of the experimental data are presented in Table 1. In addition, the typical peak hour traffic flow variables for the roadways understudy are presented in Table 2.
Noise data measured throughout this study were modeled using some independent parameters, which we think affect noise levels. These parameters are the number of vehicles, the equivalent number of cars per hour, the heavy vehicle percentage, the width of road and the average height of buildings facing the road. These 5 parameters were used as the input parameters for the neural network models. The output is the noise level measured in $\mathrm{dBA}$ as Leq at the point of measurement. The data collected throughout the study consist of a total of 620 measurement patterns. The whole database was randomly divided into 2 equal subsets; training and testing sets. The training set was used in training the networks for the best model performance. After this stage, the model was tested using the test data set to see what performance it provides when unseen data are introduced. Results of the training stage stopped at the best model point and the results of model predictions.

The actual architecture of ANN model and its parameter variation are selected based on the minimum value for the mean absolute error of the training and prediction set. The ANN architecture was optimized by the following method. Initially, the model type with activation function and with test set size was optimized. Then, the learning rate and momentum rate was varied for the optimum BPNN model. Then, the number of neurons in the hidden layers was optimized. Finally, the initial weight was examined. The optimal value of neural network parameters such as test set percentage, number of hidden layers, number of hidden neurons, initial weights, and learning and momentum rate needed to be determined for BPNN algorithm. It was important to ensure that each network learned a functional relationship between the input and output variables instead of memorizing the training examples. For this purpose, the data sets were randomly shuffled and then split into three data sets (training, testing, and prediction data sets). Six different neural network architectures were trained to find an optimum network architecture for the BPNN model. The optimum architecture was chosen based on the minimum MAE as obtained from the results of both the training and testing data sets as well as prediction data set.

Table 3 shows the MAEs obtained at the end of 10,000 training epochs from the six network architectures for five different test size percentages. The architecture prediction MAE was slightly higher than training MAE but in general was in the same order of magnitude thus indicating proper network training (Table 3). The optimum testing MAE for the two parallel hidden layers BPNN was the smallest for, however, a large number of error was encountered during prediction stage. The occurrence of this large prediction error represents a high variability in the precision of the prediction capability of the network. On the other hand, the one hidden layer showed possible overtraining displayed by the increasing training MAE. Networks with logistic and Gaussian functions were trained, tested and predicted using a back-propagation algorithm with a value of 0.1 for both learning and momentum rate.

In addition to the above parameters, the initial weights, the learning rate and momentum rate are three other important parameters that control how effectively the back-propagation algorithm trains the neural network. But for the current case, adjusting the initial weights from 0.05 to 0.3 did not affect the MAE of the training and prediction networks. At initial weight of 0.5 the MAE increased to 8 and 11.0 for training and prediction respectively. Above initial weight of 0.5 , the network error improved significantly (Table 4). Furthermore, varying the momentum rate and the learning rate from 0.05 to 1 did not affect the error of the training and prediction networks. Hence, the initial values of learning rate and momentum set by the software were used 
for all training networks. From the results of the study, the optimal configuration of ANN model was summarized in the following Table 5.

The three models described - the regression models, the BPNN, and the GRNN model were employed to predict the level of generated traffic noise at a number of study roadway locations. The results of predictions by the BPNN, GRNN and the regression models for the peak hour noise levels along with the actual measurements of traffic noise at selected roadway sites are presented in Table 6 . It can be seen from Table 8 that not all the measured noise profiles were close to the predicted noise profiles in the prediction samples. The most important benefit of the GRNN model is the large reduction in the time needed to optimize the network. For each BPNN run, computation time was high and also complicated for routine design applications. In contrast, the GRNN model requires no architecture construction or optimization. Furthermore, the genetic algorithm predicted noise in the blink of an eye. For a large number of analyses to be performed, the time saved using the GRNN model can be invaluable.

Table 6 shows the results of the GRNN model for both algorithms and verifies that the genetic algorithm is the most accurate neural network paradigm compared with the optimum BPNN model. As can be seen from Table 6 , very little improvement was achieved by switching the algorithm from genetic to iterative mode. It is clear that the genetic mode outperforms the iterative mode, and easily outperforms the BPNN model.

Two parallel hidden layer back-propagation ANN (BPNN) model was developed to predict traffic noise. Compared to GRNN architecture, BPNN model had better training and prediction fitness to experimental results. Through the prediction matrices and response plots generated by the GRNN models, there was no need for optimal processing conditions for noise profiles. ANN models could thus be effectively used for predictive modeling and optimization of noise profiles. Whilst it was possible to get a good performance with BPNN, GRNN out-performed this method somewhat. The use of the GRNN model resulted in both a drastic reduction in computation speed and simplification of parameter optimization over the BPNN model. Finally, the application of GRNN using genetic algorithm model to predict the results of noise profiles proved to be very promising and could be applied in a continuous monitoring of noise profiles.

The measured and the predicted noise levels - by the BPNN model - for the typical peak and off-peak hour at the study roadway locations are shown in Table 7. Also, shown in the table, are the differences between the measured and the predicted noise levels for each monitored roadway location. The model-predicted values are all, but one, higher than the measured noise levels. The difference between the predicted and measured noise levels during the peak hour ranges from a low of $0.9(\mathrm{dBA})$, at Gulf road to a high of $2.4(\mathrm{dBA})$ at Minawer street. A similar trend also existed for the off-peak hour noise pollution levels at the two sample districts (Table 7). An observational analysis of the predicted noise levels by the three models - the BPNN, Regressions, and the GRNN - indicates that the GRNN model and the BPNN model demonstrate slightly better predictive ability than the regression model (Table 8). The two developed disaggregate linear regression models for the peak and the off-peak hours, showed an acceptable goodness of fit and predictive ability. These models generally slightly overestimated traffic noise pollution levels (Table 9).

\section{Conclusions}

1. The two developed disaggregate linear regression models for the peak and the off-peak hours, showed an acceptable goodness of fit and

\begin{tabular}{|l|l|l|l|l|l|l|}
\hline \multirow{2}{*}{$\begin{array}{l}\text { District/ } \\
\text { Roadway }\end{array}$} & \multicolumn{5}{|c|}{ Noise Level (dBA) } \\
\cline { 2 - 7 } & \multicolumn{3}{|c|}{ Peak Hour } & \multicolumn{3}{|c|}{ Off-Peak Hour } \\
\cline { 2 - 7 } & Measured & Predicted & Difference & Measured & Predicted & Difference \\
\hline Gulf Rd. & 81.4 & 80.5 & -0.9 & 74.0 & 78.5 & +4.5 \\
S. Mobarak & 75.3 & 77.5 & +2.2 & 76.1 & 77.5 & +1.4 \\
\hline Ghazali & 80.2 & 82.4 & +2.2 & 79.5 & 77.3 & -2.2 \\
Minawer & 72.0 & 74.4 & +2.4 & 72.1 & 74.1 & +2.0 \\
\hline
\end{tabular}

Table 7: Comparison of Measured and BPNN-Predicted Noise Levels.

\begin{tabular}{|l|l|l|l|l|l|l|}
\hline \multirow{2}{*}{$\begin{array}{l}\text { District/ } \\
\text { Roadway }\end{array}$} & \multicolumn{5}{|c|}{ Noise Level (dBA) } \\
\cline { 2 - 7 } & \multicolumn{4}{|c|}{ Peak Hour } & \multicolumn{3}{c|}{ Off-Peak Hour } \\
\cline { 2 - 7 } & Measured & Predicted & Difference & Measured & Predicted & Difference \\
\hline Gulf Rd. & 81.4 & 81.5 & +0.1 & 74.0 & 74.5 & +0.5 \\
S. Mobarak & 75.3 & 75.5 & +0.2 & 76.1 & 76.5 & +0.4 \\
\hline Ghazali & 80.2 & 80.3 & +0.1 & 79.5 & 79.7 & +0.2 \\
Minawer & 72.0 & 72.2 & +0.2 & 72.1 & 72.1 & 0 \\
\hline
\end{tabular}

Table 8: Comparison of Measured and GRNN-Predicted Noise Levels.

\begin{tabular}{|l|l|l|l|l|l|l|}
\hline \multirow{2}{*}{$\begin{array}{l}\text { District/ } \\
\text { Roadway }\end{array}$} & \multicolumn{5}{|c|}{ Noise Level (dBA) } \\
\cline { 2 - 7 } & \multicolumn{3}{|c|}{ Peak Hour } & \multicolumn{3}{c|}{ Off-Peak Hour } \\
\cline { 2 - 7 } & Measured & Predicted & Difference & Measured & Predicted & Difference \\
\hline Gulf Rd. & 81.4 & 84.5 & +3.1 & 74.0 & 81.5 & +7.5 \\
S. Mobarak & 75.3 & 79.5 & +4.2 & 76.1 & 87.5 & +11.4 \\
\hline Ghazali & 80.2 & 85.5 & +5.3 & 79.5 & 86.3 & +6.8 \\
Minawer & 72.0 & 79.0 & +7.0 & 72.1 & 79.1 & +7.0 \\
\hline
\end{tabular}

Table 9: Comparison of Measured and Regression-Predicted Noise Levels.

predictive ability. These models generally slightly overestimated traffic noise pollution levels.

2. The BPNN model generally overestimated traffic noise levels at the study urban roadways and streets in Kuwait. The GRNN model also predicted the noise levels rather accurately, usually overestimating the levels of noise from 0.1 to $0.6 \mathrm{dBA}$.

3. The nonlinear ANN model identified using the GRNN identification procedure seems to provide a better systems theoretical representation of the noise prediction than the BPNN model. In addition, the performance of the nonlinear ANN models appears to be superior to that of the conceptual regression model. Because the ANN approach presented here does not provide models that have physically realistic components and parameters, it is by no means a substitute for conceptual traffic noise modeling. However, the results suggest that the ANN approach may provide a superior alternative to the regression-series approach for developing input-output simulation and forecasting models in situations that do not require modeling.

\section{References}

1. Barry T, Reagan J (1978) FHWA Highway Traffic Noise Prediction Model Report No. FHWA-RD-77-108, Federal Highway Administration, Washington DC.

2. Jakovljevic B, Paunovic K, Belojevic G (2009) Road-traffic noise and factors influencing noise annoyance in an urban population. Environ Int 35: 552-556.

3. Givargis Sh, Karimi H (2010) A basic neural traffic noise prediction model for Tehran's roads. J Environ Manage 91: 2529-2534.

4. Cammarata G, Cavalieri S, Fichera A (1995) A neural network architecture for noise prediction. Neural Networks 8: 963-973.

5. Genaro N, Torija A, Ramos A, Requena I, Ruiz DP, et al. (2009) Modeling Environmental Noise Using Artificial Neural Networks. Ninth International Conference on Intelligent Systems Design and Application 215-219.

6. Givargis Sh, Karimi H (2009) Mathematical, statistical and neural models capable of predicting $\mathrm{L}_{\text {Amax }}$ for the Tehran-Karaj express train. Applied Acoustics 70: 1015-1020.

7. Werbos PJ (1974) Beyond regression: new tools for prediction and analysis in the behavioral sciences. Harvard University, Boston, M.A. 
8. Patterson D (1996) Artificial Neural Networks. Singapore: Prentice Hall.

9. Gundogdu O, Gokdag M, Yuksel F (2005) A traffic noise prediction method based on vehicle composition using genetic algorithms. Applied Acoustics 66: 799-809.

10. Specht DF (1991) A General Regression Neural Network. IEEE Trans Neu Net 2: $568-576$.

11. Specht DF (1993) The General Regression Neural Network-rediscovered. Neural Networks 6: 1033-1034.

12. Specht DF, Ramshahl H (1994) Experience with Adaptive probabilistic Neural Networks and Adaptive General Regression Neural networks. Proc IEEE World Congr Comp Int 2: 1203-1208.

13. Hansen JV, Meservy RD (1996) Learning experiments with genetic optimization of a Generalized Regression Neural Networks. Decision Support Systems 18: 317-325
14. Bruel and Kjear (2004) Predictor type 7810, version 2.0. User's Manual for the predictor platform, Naerum, Denmark.

15. (2001) SAS User's Guide: Statistics version ( $8^{\text {th }}$ edn). SAS Institute, Inc, Cary NC.

16. Fausett $L$ (1994) Fundamentals of neural networks: Architectures, Algorithms and application. Prentice-Hall, New Jersey, USA

17. Rumelhart DE, Hinton G, Williams R (1986) Learning Representations by backpropagating errors.

18. Masters T (1995) Advanced algorithms for neural networks: a C++ sourcebook John Wiley and Sons, New York.

19. Rahmani S, Mousavi S, Kamali M (2011) Modeling of road-traffic noise with the use of genetic algorithm. Applied Soft computing 11: 1008-1013. 